\begin{tabular}{|c|c|}
\hline Title & Finite element analysis of magnetostatic wave propagation in a Y IG film of finite dimensions \\
\hline Author(s) & Koshiba, M.; Long, Y. \\
\hline Citation & $\begin{array}{l}\text { IEEE Transactions on Microwave Theory and Techniques, 37(11), 1768-1772 } \\
\text { https://doi.org/10.1109/22.41043 }\end{array}$ \\
\hline Issue Date & $1989-11$ \\
\hline Doc URL & http:/hdl.handle.net/2115/6072 \\
\hline Rights & $\begin{array}{l}\text { ( } 1989 \text { IEEE. Personal use of this material is permitted. However, permission to reprint/republish this material for } \\
\text { advertising or promotional purposes or for creating new collective works for resale or redistribution to servers or lists, } \\
\text { or to reuse any copyrighted component of this work in other works must be obtained from the IEEE." } \\
\text { IEEE, IEEE Transactions on Microwave Theory and Techniques, volume 37, issue 11, 1989, Page(s):1768- } 1772\end{array}$ \\
\hline Tyре & article \\
\hline File Information & ITMTT37-11.pdf \\
\hline
\end{tabular}

Instructions for use 


\title{
Finite-Element Analysis of Magnetostatic Wave Propagation in a YIG Film of Finite Dimensions
}

\author{
MASANORI KOSHIBA, SENIOR MEMBER, IEEE, AND YI LONG
}

\begin{abstract}
A unified numerical approach based on the finite-element method is described for the magnetostatic wave propagation in a YIG film of finite dimensions. Both magnetostatic volume wave and magnetostatic surface wave modes are treated. The validity of the method is confirmed by calculating the magnetostatic wave modes in a YIG-loaded rectangular waveguide and in a YIG film of finite width. The numerical results of a YIG film with nonuniform bias field along the film width are also presented, and the effects of bias field distributions on the delay characteristics and potential profiles are examined.
\end{abstract}

\section{INTRODUCTION}

$\mathrm{M}$ AGNETOSTATIC WAVE (MSW) propagation in a YIG film of finite dimensions has been reported in previous papers [1]-[11]. O'Keeffe et al. [2] and Bajpai et al. [4] have investigated the effects of finite sample widths on MSW propagation. Recently, interest in threedimensional inhomogeneous MSW waveguides is increasing because high-precision dispersion control is making MSW device application possible [1], [3], [5]-[11]. Morgenthaler et al. [1], [3], [6], [8], [9] have discussed the control of MSW propagation by means of a spatially nonuniform bias field. It is also found that control of important features of MSW modes is afforded through the use of bias field gradients and that magnetostatic forward volume waves can be forced to have strong field-displacement characteristics that are either nearly reciprocal or very strongly nonreciprocal. Such control may provide the basis for new forms of microwave signal processors [6]-[9]. In order to analyze these inhomogeneous MSW waveguides, the variational method [12], [13] and the finite-element method [14] have been introduced. These methods are valid for the solution of inhomogeneous waveguide structures. In the former approach, however, great care is necessary in choosing the trial functions. The latter approach, on the other hand, is applied only to planar structures of infinite width.

In this paper, a unified approach based on the finite-element method is described for the MSW propagation in a YIG film of finite dimensions. Both magnetostatic forward volume wave (MSFVW) and magnetostatic surface wave (MSSW) modes are treated. In this finite-element ap-

Manuscript received February 23, 1989; revised July 10, 1989.

The authors are with the Department of Electronic Engineering, Hokkaido University, Sapporo, 060 Japan.

IEEE Log Number 8930663.

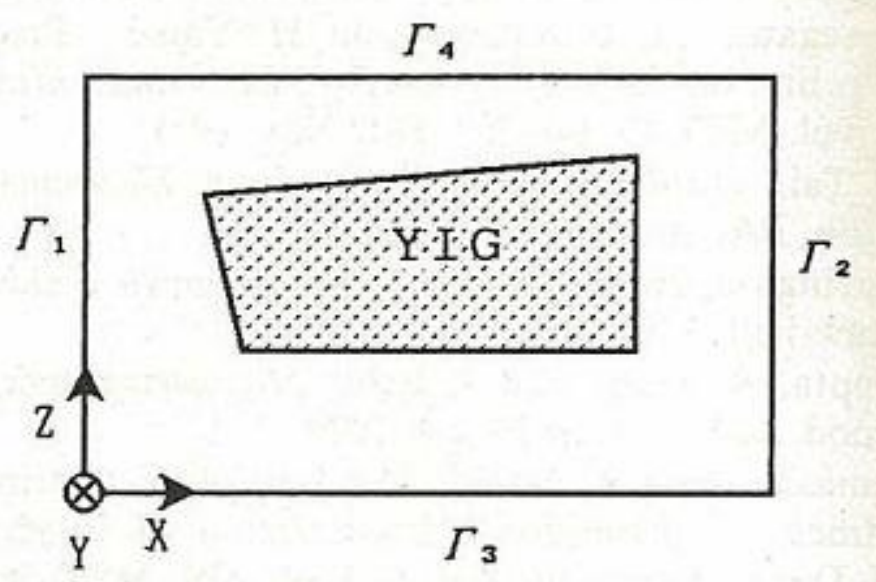

Fig. 1. MSW waveguide.

proach, the cross section of the waveguide is divided into triangular elements [15], [16] and it is easy to consider the inhomogeneities in the bias field and/or the magnetization. The validity of the method is confirmed by calculating the MSW modes in a YIG-loaded rectangular waveguide [10] and in a YIG film of finite width [4]. Numerical results on the delay characteristics and potential profiles of a YIG film with nonuniform bias field distributions are examined.

\section{Basic EQUATIONS}

Fig. 1 shows a MSW waveguide, where the boundaries $\Gamma_{1}$ and $\Gamma_{2}$ are assumed to be perfect electric conductors (PEC's) or perfect magnetic conductors (PMC's), and $\Gamma_{3}$ and $\Gamma_{4}$ are assumed to be PEC's.

The constitutive relations are

$$
\begin{array}{lll}
\boldsymbol{B}=\mu_{0}\left[\mu_{r}\right] \boldsymbol{H} & \text { for ferrite } \\
\boldsymbol{B}=\mu_{0} \boldsymbol{H} & \text { for dielectric }
\end{array}
$$

where $\boldsymbol{B}$ is the magnetic flux density, $\boldsymbol{H}$ is the magnetic field, $\mu_{0}$ is the permeability in free space, and $\left[\mu_{r}\right]$ is the relative permeability tensor [14].

For the MSW propagating along the $y$ direction, the governing equations can be written by

$$
\begin{gathered}
\frac{\partial B_{x}}{\partial x}+\frac{\partial B_{z}}{\partial z}-j s \beta B_{y}=0 \\
H_{x}=-\frac{\partial \phi}{\partial x} \\
H_{y}=j s \beta \phi \\
H_{z}=-\frac{\partial \phi}{\partial z}
\end{gathered}
$$




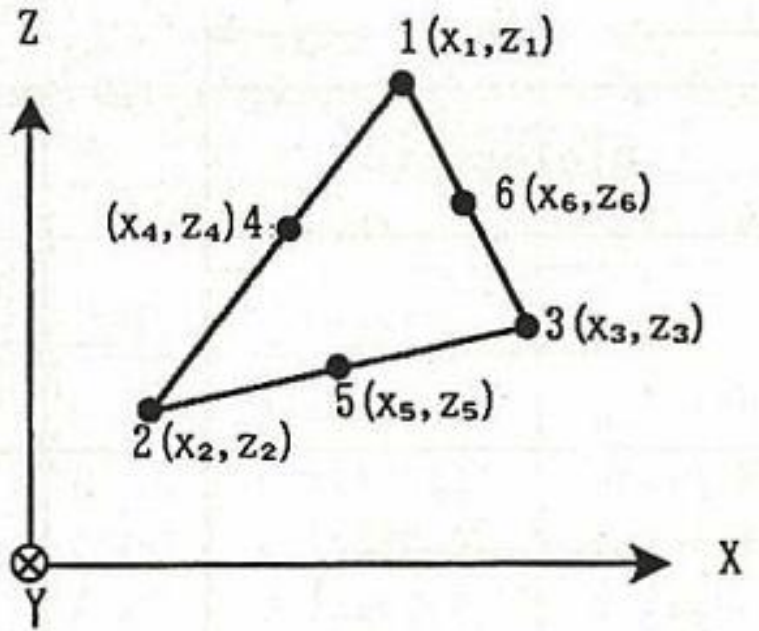

Fig. 2. Second-order triangular element.

where $\beta$ is the phase constant in the $y$ direction, $s= \pm 1$ is a directional parameter [14], and $\phi$ is the magnetostatic potential.

\section{Mathematical Formulations}

Dividing the region enclosed by the boundaries $\Gamma_{1}$ to $\Gamma_{4}$ into a number of second-order triangular elements [15], $[16]$ as shown in Fig. 2, the magnetic potential $\phi$ within each element is defined in terms of the magnetic potential $\phi_{k}$ at the nodal point $k(k=1,2, \cdots, 6)$ :

$$
\phi=\{N\}^{T}\{\phi\}_{e} \exp \left(-j s \beta_{y}\right)
$$

where

$$
\begin{aligned}
& \{\phi\}_{e}=\left[\begin{array}{llllll}
\phi_{1} & \phi_{2} & \phi_{3} & \phi_{4} & \phi_{5} & \phi_{6}
\end{array}\right]^{T} \\
& \{N\}=\left[\begin{array}{llllll}
N_{1} & N_{2} & N_{3} & N_{4} & N_{5} & N_{6}
\end{array}\right]^{T} .
\end{aligned}
$$

Here shape functions $N_{1}$ to $N_{6}$ are given by

$$
\begin{aligned}
& N_{1}=L_{1}\left(2 L_{1}-1\right) \\
& N_{2}=L_{2}\left(2 L_{2}-1\right) \\
& N_{3}=L_{3}\left(2 L_{3}-1\right) \\
& N_{4}=4 L_{1} L_{2} \\
& N_{5}=4 L_{2} L_{3} \\
& N_{6}=4 L_{3} L_{1} .
\end{aligned}
$$

with the area coordinates $L_{1}, L_{2}$, and $L_{3}$ [15], [16]. The relation between the area coordinates and the Cartesian coordinates is expressed in the following form:

$$
\left[\begin{array}{l}
x \\
z \\
1
\end{array}\right]=\left[\begin{array}{ccc}
x_{1} & x_{2} & x_{3} \\
z_{1} & z_{2} & z_{3} \\
1 & 1 & 1
\end{array}\right]\left[\begin{array}{l}
L_{1} \\
L_{2} \\
L_{3}
\end{array}\right]
$$

where $\left(x_{j}, z_{j}\right)$ are the Cartesian coordinates of the vertex $j$ $(j=1,2,3)$ of the triangle. Also, the diagonal component $\mu$ and the off-diagonal component $\kappa$ of the relative permeability tensor within each element are approximated by

$$
\begin{aligned}
& \mu=\{N\}^{T}\{\mu\}_{e} \\
& \kappa=\{N\}^{T}\{\kappa\}_{e}
\end{aligned}
$$

where

$$
\begin{aligned}
& \{\mu\}_{e}=\left[\begin{array}{llllll}
\mu_{1} & \mu_{2} & \mu_{3} & \mu_{4} & \mu_{5} & \mu_{6}
\end{array}\right]^{T} \\
& \{\kappa\}_{e}=\left[\begin{array}{llllll}
\kappa_{1} & \kappa_{2} & \kappa_{3} & \kappa_{4} & \kappa_{5} & \kappa_{6}
\end{array}\right]^{T}
\end{aligned}
$$

and $\mu_{k}$ and $\kappa_{k}(k=1,2, \cdots, 6)$ are, respectively, the $\mu$ and $\kappa$ values at the nodal point $k$. Using a Galerkin procedure on (2), we obtain

$$
\int_{e}\{N\}\left(\frac{\partial B_{x}}{\partial x}+\frac{\partial B_{z}}{\partial z}-j s \beta B_{y}\right) d \Omega=\{0\}
$$

where the integration is carried out over the element subdomain $\Omega_{e}$, and $\{0\}$ is a null vector.

Integrating by parts, we obtain for (10)

$$
\begin{aligned}
\int_{e}\left[\left\{N_{x}\right\} B_{x}+\left\{N_{z}\right\} B_{z}+j s \beta\{N\} B_{y}\right] d \Omega \\
\\
\quad-\int_{e}\{N\} B_{n} d \Gamma=\{0\}
\end{aligned}
$$

where

$$
B_{n}=B_{x} n_{x}+B_{z} n_{z} .
$$

Here $\left\{N_{x}\right\}=\partial\{N\} / \partial x ;\left\{N_{z}\right\}=\partial\{N\} / \partial z$; the second integration on the left-hand side in (11) is carried out over the contour $\Gamma_{e}$ of the region $\Omega_{e}$; and $n_{x}$ and $n_{z}$ are the $x$ and $z$ components of an outward normal unit vector to $\Gamma_{e}$, respectively.

Noting that $B_{n}$ is continuous across $\Gamma_{e}$ (boundary conditions at the interface between two different media) and $B_{n}=0$ on $\Gamma_{3}$ and $\Gamma_{4}$, from (1), (3), and (4) the following global matrix equation is derived:

$$
[A]\{\phi\}=-\sum_{i=1}^{2}(-1)^{i} \sum_{e^{\prime}} \int_{e^{\prime}}\left[\{N\}_{i} B_{x, i}\right] / \mu_{0} d z
$$

where

$$
\begin{aligned}
{[A]=\sum_{e} \int_{e}\left[\beta^{2} \mu_{e}\{N\}\{N\}^{T}-\beta s \kappa_{e}\right.} \\
\cdot\left(\left\{N_{z}\right\}\{N\}^{T}+\{N\}\left\{N_{z}\right\}^{T}\right) \\
\left.+\left\{N_{x}\right\}\left\{N_{x}\right\}^{T}+\mu_{e}\left\{N_{z}\right\}\left\{N_{z}\right\}^{T}\right] d x d z
\end{aligned}
$$

for bias field applied parallel to the $x$ axis

$$
\begin{aligned}
{[A]=\sum_{e} \int_{e}[} & \beta^{2}\{N\}\{N\}^{T}+j \kappa_{e} \\
& \cdot\left(\left\{N_{z}\right\}\left\{N_{x}\right\}^{T}-\left\{N_{x}\right\}\left\{N_{z}\right\}^{T}\right) \\
& \left.+\mu_{e}\left(\left\{N_{x}\right\}\left\{N_{x}\right\}^{T}+\left\{N_{z}\right\}\left\{N_{z}\right\}^{T}\right)\right] d x d z
\end{aligned}
$$

for bias field applied parallel to the $y$ axis

and

$$
\begin{aligned}
{[A]=\sum_{e} \int_{e}\left[\beta^{2} \mu_{e}\{N\}\{N\}^{T}+\beta s \kappa_{e}\right.} \\
\cdot\left(\left\{N_{x}\right\}\{N\}^{T}+\{N\}\left\{N_{x}\right\}^{T}\right) \\
\left.+\left\{N_{z}\right\}\left\{N_{z}\right\}^{T}+\mu_{e}\left\{N_{x}\right\}\left\{N_{x}\right\}^{T}\right] d x d z
\end{aligned}
$$

for bias field applied parallel to the $z$ axis.

Here $\mu_{e}$ and $\kappa_{e}$ are expressed by (8a) and (8b), respectively; $\{\phi\}$ is the nodal magnetic potential; $\sum_{e}$ and $\sum_{e^{\prime}}$ 
extend over all elements and the elements related to $\Gamma_{i}$ $(i=1,2)$, respectively; $B_{x, i}$ is the magnetic flux density on $\Gamma_{i}$; and $\{N\}_{i}$ is the shape function vector on $\Gamma_{i}$, namely, $\{N\}_{i}=\left\{N\left(x_{i}, z\right)\right\}$.

Combining the boundary conditions on the planes $\Gamma_{1}$ and $\Gamma_{2},(13)$ becomes

$$
[\tilde{A}]\{\tilde{\phi}\}=\{0\}
$$

where

$$
[A]= \begin{cases}{[A]} & \text { for } B_{x, 1}=B_{x, 2}=0 \\
{[A]_{00}} & \text { for }\{\phi\}_{1}=\{\phi\}_{2}=\{0\} \\
{\left[\begin{array}{ll}
{[A]_{00}} & {[A]_{01}} \\
{[A]_{10}} & {[A]_{11}}
\end{array}\right]} & \text { for } B_{x, 1}=0 \text { and }\{\phi\}_{2}=\{0\} \\
{\left[\begin{array}{ll}
{[A]_{00}} & {[A]_{02}} \\
{[A]_{20}} & {[A]_{22}}
\end{array}\right]} & \text { for } B_{x, 2}=0 \text { and }\{\phi\}_{1}=\{0\}\end{cases}
$$

$$
\begin{aligned}
& \{\tilde{\phi}\}= \begin{cases}\{\phi\} & \text { for } B_{x, 1}=B_{x, 2}=0 \\
\{\phi\}_{0} & \text { for }\{\phi\}_{1}=\{\phi\}_{2}=\{0\} \\
{\left[\begin{array}{l}
\{\phi\}_{0} \\
\{\phi\}_{1}
\end{array}\right]} & \text { for } B_{x, 1}=0 \text { and }\{\phi\}_{2}=\{0\} \\
{\left[\begin{array}{l}
\{\phi\}_{0} \\
\{\phi\}_{2}
\end{array}\right]} & \text { for } B_{x, 2}=0 \text { and }\{\phi\}_{1}=\{0\}\end{cases} \\
& \{\phi\}=\left[\begin{array}{l}
\{\phi\}_{0} \\
\{\phi\}_{1} \\
\{\phi\}_{2}
\end{array}\right] \\
& {[A]=\left[\begin{array}{lll}
{[A]_{00}} & {[A]_{01}} & {[A]_{02}} \\
{[A]_{10}} & {[A]_{11}} & {[A]_{12}} \\
{[A]_{20}} & {[A]_{21}} & {[A]_{22}}
\end{array}\right] .}
\end{aligned}
$$

Here $[A]_{00},[A]_{01}, \cdots,[A]_{22}$ are the submatrices of $[A]$, and $\{\phi\}_{i}$ is the nodal magnetic potential on $\Gamma_{i}(i=1,2)$. Using (15), one can determine the dispersion characteristics of MSW waveguides.

\section{Computed Results}

First, we consider a YIG-loaded rectangular waveguide [10] as shown in Fig. 3, where the planes $x=0, x=a$, $z=0$, and $z=b$ are assumed to be PEC's, the bias field is along the $x$ axis, and there exist MSSW modes. In this case, the potential profile is symmetric with respect to the plane $x=a / 2$. Taking advantage of this symmetry, we subdivided one half of the cross section into second-order triangular elements, where the plane $x=a / 2$ is assumed to be a PMC. Table I exhibits the computed results, where $a=20 \mathrm{~mm}, b=10 \mathrm{~mm}, t=1 \mathrm{~mm}$, the saturation magnetization $4 \pi M_{s}=1750 \mathrm{G}$, the bias field $H_{0}=1800 \mathrm{Oe}, \beta_{\text {exact }}$ is the exact solution [10], and $\beta_{\mathrm{FEM}}$ is the finite-element solution. It is apparent from Table I that the finite-element solutions are in good agreement with the exact solutions. The convergence behavior of the phase constant $\beta$ is shown in Fig. 4. With an increasing number of elements, the phase constant $\beta$ converges monotonically. Also, we

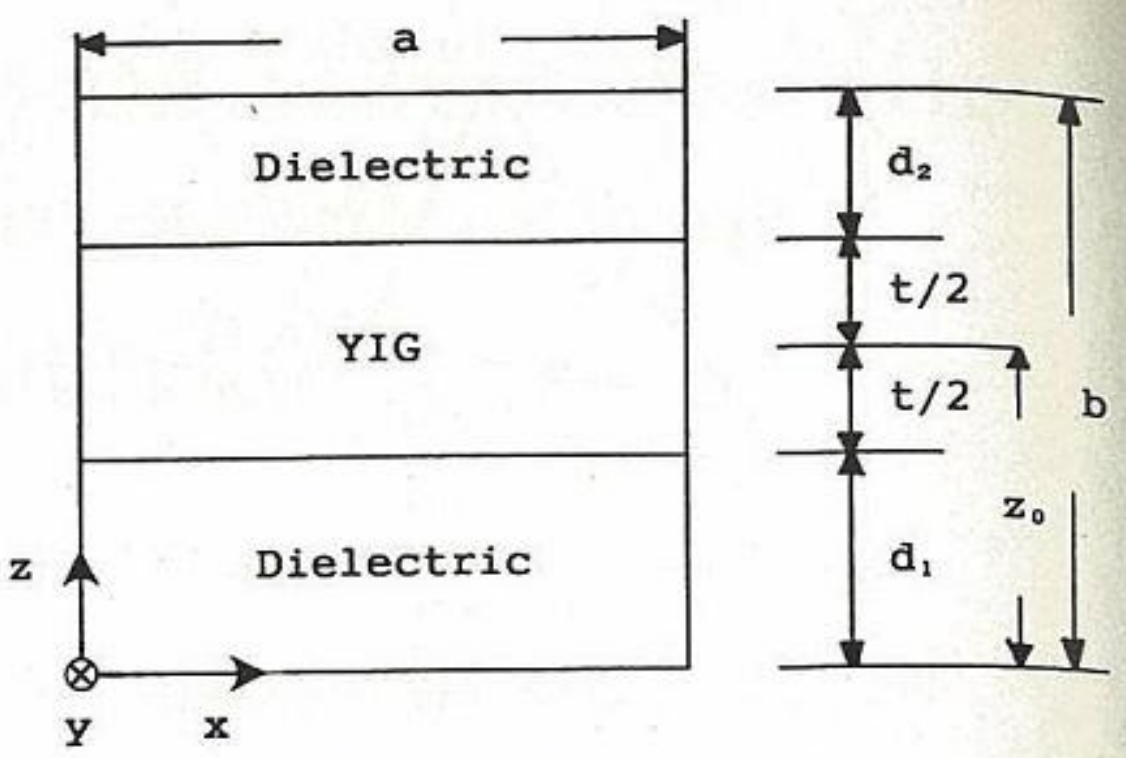

Fig. 3. A YIG-loaded rectangular waveguide (the planes $x=0, x=0$ $z=0$, and $z=b$ are assumed to be PEC's) or a YIG film of finite width (the planes $x=0$ and $x=\mathrm{a}$ are assumed to be PMC's and the planes $z=0$ and $z=\mathrm{b}$ are assumed to be PEC's).

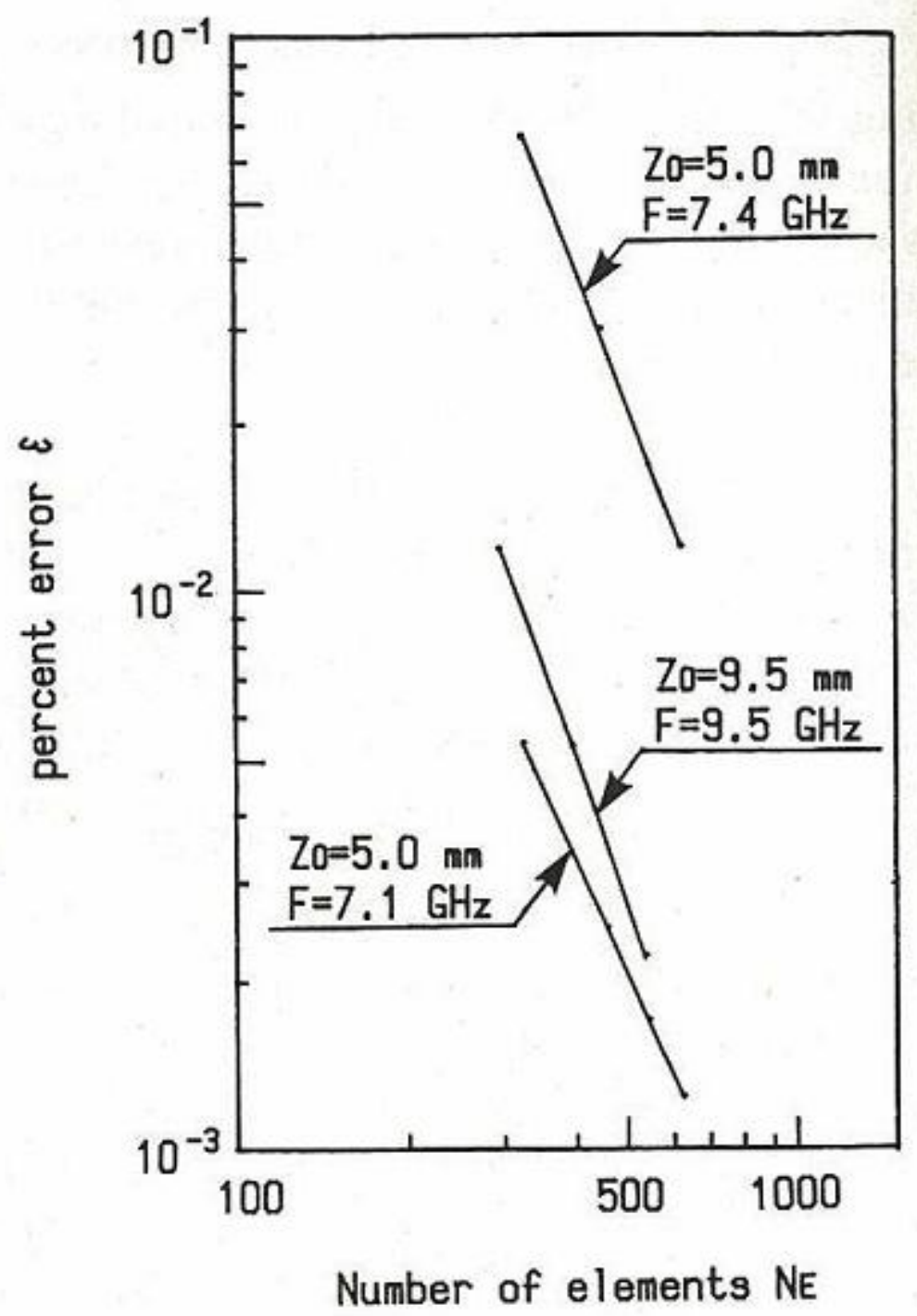

Fig. 4. Convergence behavior of phase constant $\beta$.

find from Table I and Fig. 4 that the accuracy of the finite-element solutions falls off as the film position $z_{0}$ is lowered or the frequency $F$ increases.

Next, we consider a YIG film of finite width [4] as shown in Fig. 3, where the planes $x=0$ and $x=a$ are assumed to be PMC's, the planes $z=0$ and $z=b$ are assumed to be PEC's [2], [4], the bias field is along the $z$ axis, and there exist MSFVW modes. Table II exhibits the computed results, where $a=3 \mathrm{~mm}, d_{1}=50 \mu \mathrm{m}, d_{2}=178$ $\mu \mathrm{m}, t=9.1 \mu \mathrm{m}, 4 \pi M_{s}=1750 \mathrm{G}$, and the effective static field $H_{i}=3000 \mathrm{Oe}\left(H_{i}=H_{0}-4 \pi M_{s}\right.$ for MSFVW modes). The finite-element solutions agree well with the exact solutions [4]. Also, larger errors are observed as the frequency increases.

Lastly, we consider the MSW waveguide with a bias field of parabolic distribution where the planes $x=0$ and $x=a$ in Fig. 3 are assumed to be PMC's, the planes $z=0$ and $z=b$ are assumed to be PEC's, and the bias field is 
TABLE I

COMPaRison Between EXACT SOlutions AND FEM SOLUTIONS FOR MSSW MODES

\begin{tabular}{c|c|c|c}
\hline \hline \multirow{2}{*}{$z_{0}$} & $F$ & \multicolumn{2}{|c}{ Phase constant $(1 / \mathrm{mm})$} \\
\cline { 3 - 4 }$(\mathrm{mm})$ & $(\mathrm{GHz})$ & $\beta_{\text {exact }}$ & $\beta_{\text {FEM }}$ \\
\hline & 7.1 & 0.0419056 & 0.0419052 \\
& 7.3 & 0.1193626 & 0.1193620 \\
8.0 & 7.4 & 0.1633299 & 0.1633295 \\
& 7.5 & 0.2235310 & 0.2235304 \\
& 7.6 & 0.3563842 & 0.3563856 \\
\hline \multirow{5}{*}{5.0} & 7.1 & 0.1104845 & 0.1104839 \\
& 7.2 & 0.2253471 & 0.2253469 \\
& 7.3 & 0.4832040 & 0.4832315 \\
& 7.4 & 0.9011406 & 0.9019049 \\
\hline
\end{tabular}

$a=20 \mathrm{~mm}, b=10 \mathrm{~mm}, t=1 \mathrm{~mm}, 4 \pi M_{s}=1750 \mathrm{G}$, $H_{0}=1800 \mathrm{Oe}$.

TABLE II

COMPARISON BETWEen EXACT SOlutions AND FEM SOLUTIONS FOR MSFVW MODES

\begin{tabular}{c|c|c}
\hline \hline \multirow{2}{*}{\begin{tabular}{c} 
(GHz) \\
\cline { 2 - 3 }
\end{tabular}} & \multicolumn{2}{|c}{ Phase constant $(1 / \mathrm{mm})$} \\
\cline { 2 - 3 } 8.81 & 3.200592 & 3.200420 \\
8.91 & 16.25167 & 16.25179 \\
9.01 & 28.59313 & 28.59392 \\
9.11 & 41.05768 & 41.06009 \\
9.21 & 53.96476 & 53.97119 \\
9.31 & 67.73479 & 67.74865 \\
9.41 & 82.69378 & 82.71970 \\
9.51 & 99.12766 & 99.17226 \\
\hline
\end{tabular}

$d_{1}=50 \mu \mathrm{m}, d_{2}=178 \mu \mathrm{m}, t=9.1 \mu \mathrm{m}$, $a=3 \mathrm{~mm}, 4 \pi M_{s}=1750 \mathrm{G}$, and $H_{i}=$ $3000 \mathrm{O}$.

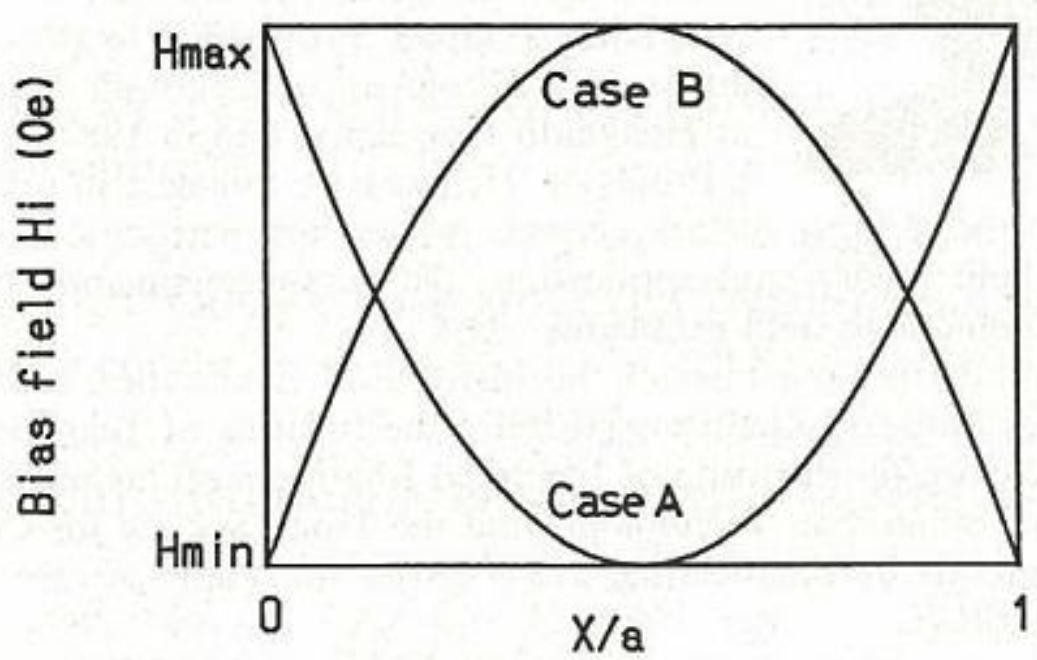

Fig. 5. Bias field distribution along the width of a YIG film.

along the $z$ axis. The two parabolic distributions considered here are shown in Fig. 5. The bias field distribution is given by

$$
H_{i}=H_{1}-\left(H_{1}-H_{2}\right) \times[(a-2 x) / a]^{2}
$$

where $H_{1}$ and $H_{2}$ are the minimum and the maximum value of the effective field for a hollowed parabolic distribution (case A), respectively, and $H_{1}$ and $H_{2}$ are the maximum and the minimum value for a projecting parabolic distribution (case B), respectively.

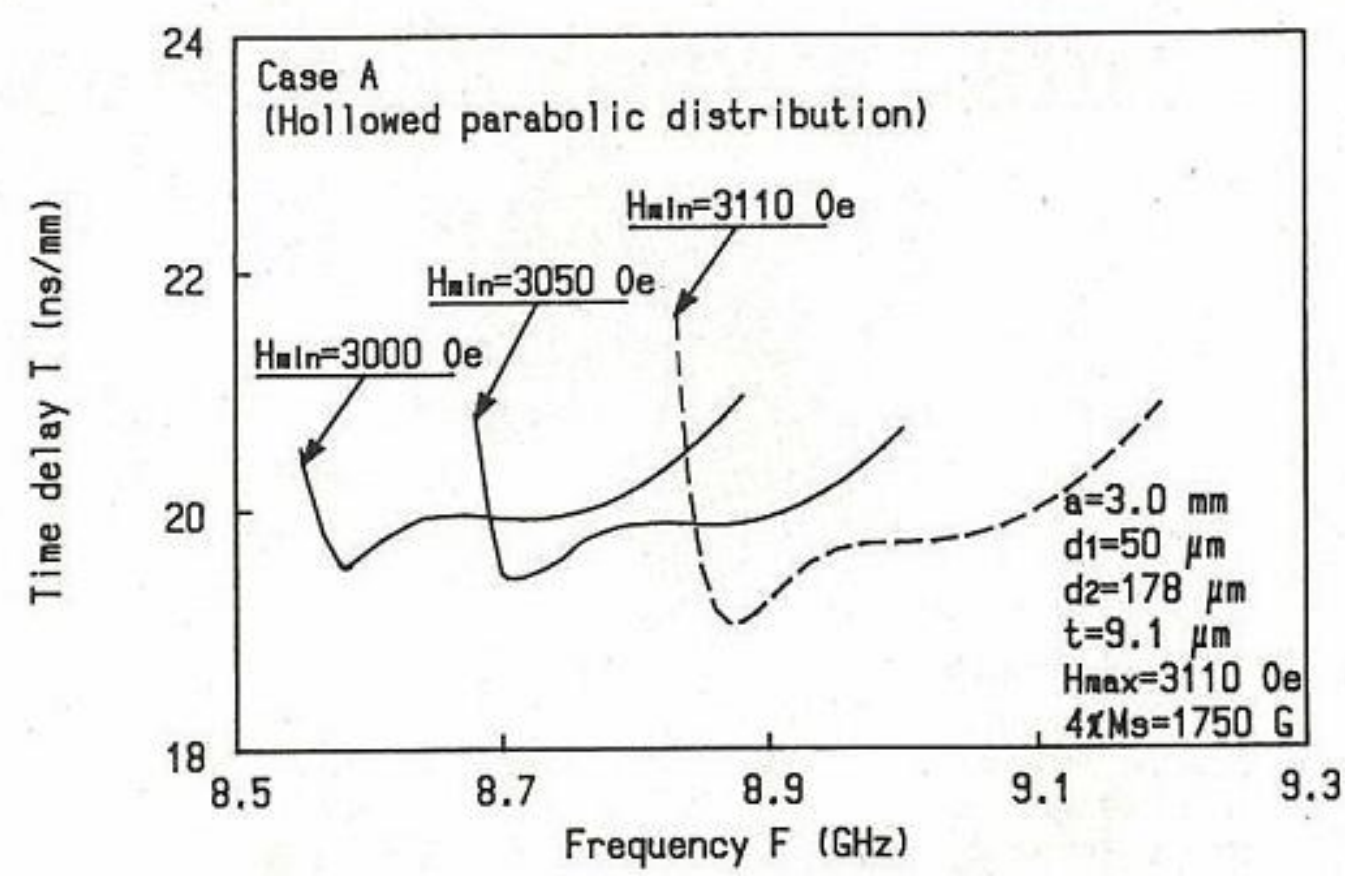

(a)

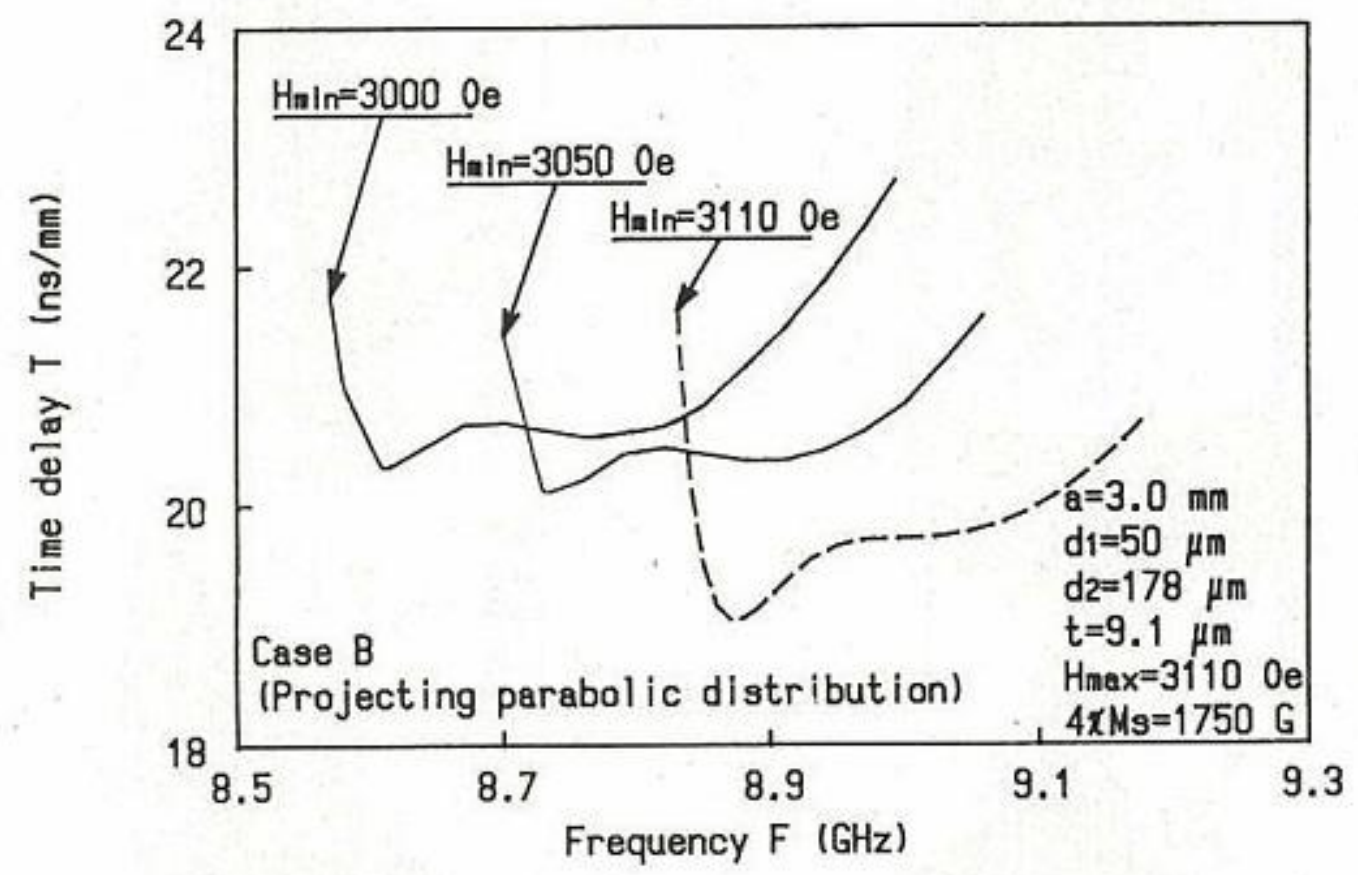

(b)

Fig. 6. Time delay characteristics for parabolic bias field distribution along the film width. (a) Hollowed parabolic distribution. (b) Projecting parabolic distribution.

The time delay characteristics are illustrated in Fig. 6(a) and (b) for cases A and B, respectively, where the dotted line represents the delay curve for the homogeneous case [4]. These figures show that the nondispersive bandwidth is extended in the case of the parabolic distribution of the bias field. Comparing case A with case B, it is also found that the projecting parabolic bias field offers a wider nondispersive bandwidth.

The effect of parabolic bias field on the potential profile is investigated, Fig. 7(a), (b), and (c) shows the potential profiles for the uniform, hollowed parabolic, and projecting parabolic bias fields, respectively. The phase constant $\beta$ is independent of the direction of propagation. In the case of parabolic bias fields, the potential has a stronger localization. Comparing case A with case B, it is found that the projecting parabolic bias field creates stronger potential-displacement nonreciprocity.

A similar potential-displacement nonreciprocity was reported in [9].

\section{CONCLUSIONS}

A finite-element method is developed for the analysis of the MSW propagation in a YIG film of finite dimensions. Both MSVW and MSSW modes are treated in a unified 


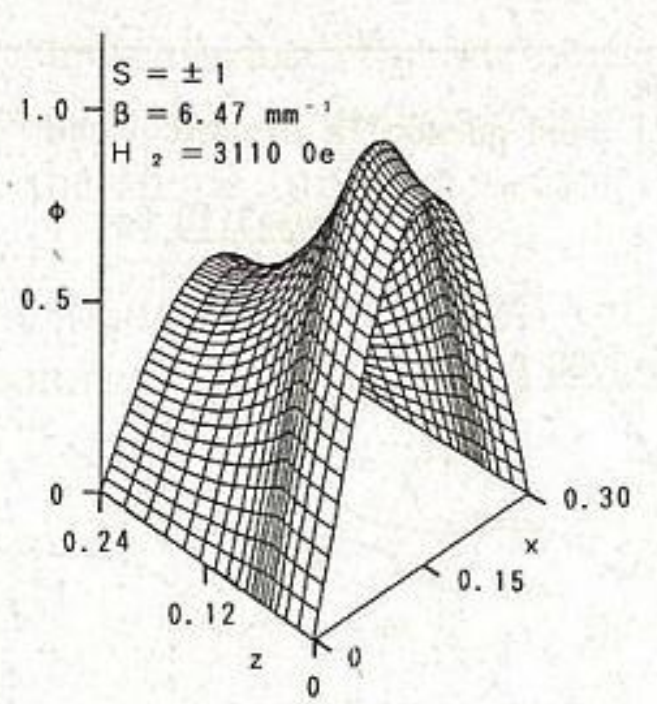

(a)

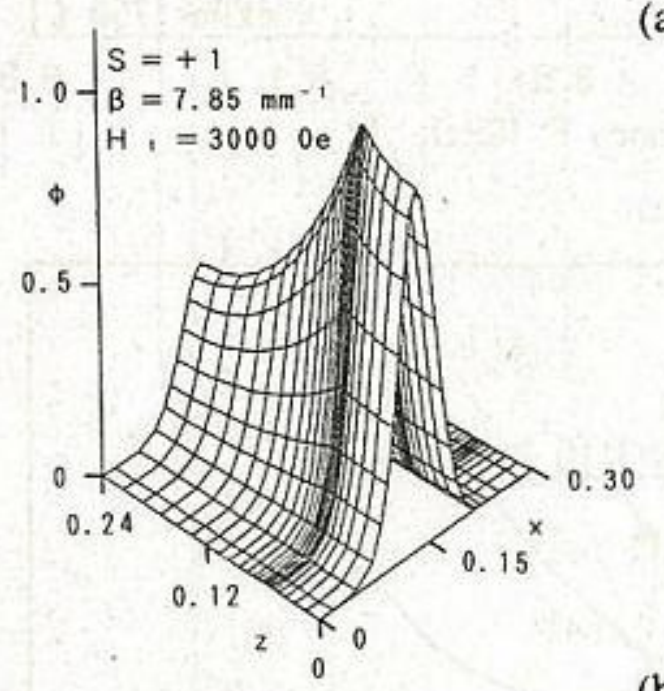

(b)
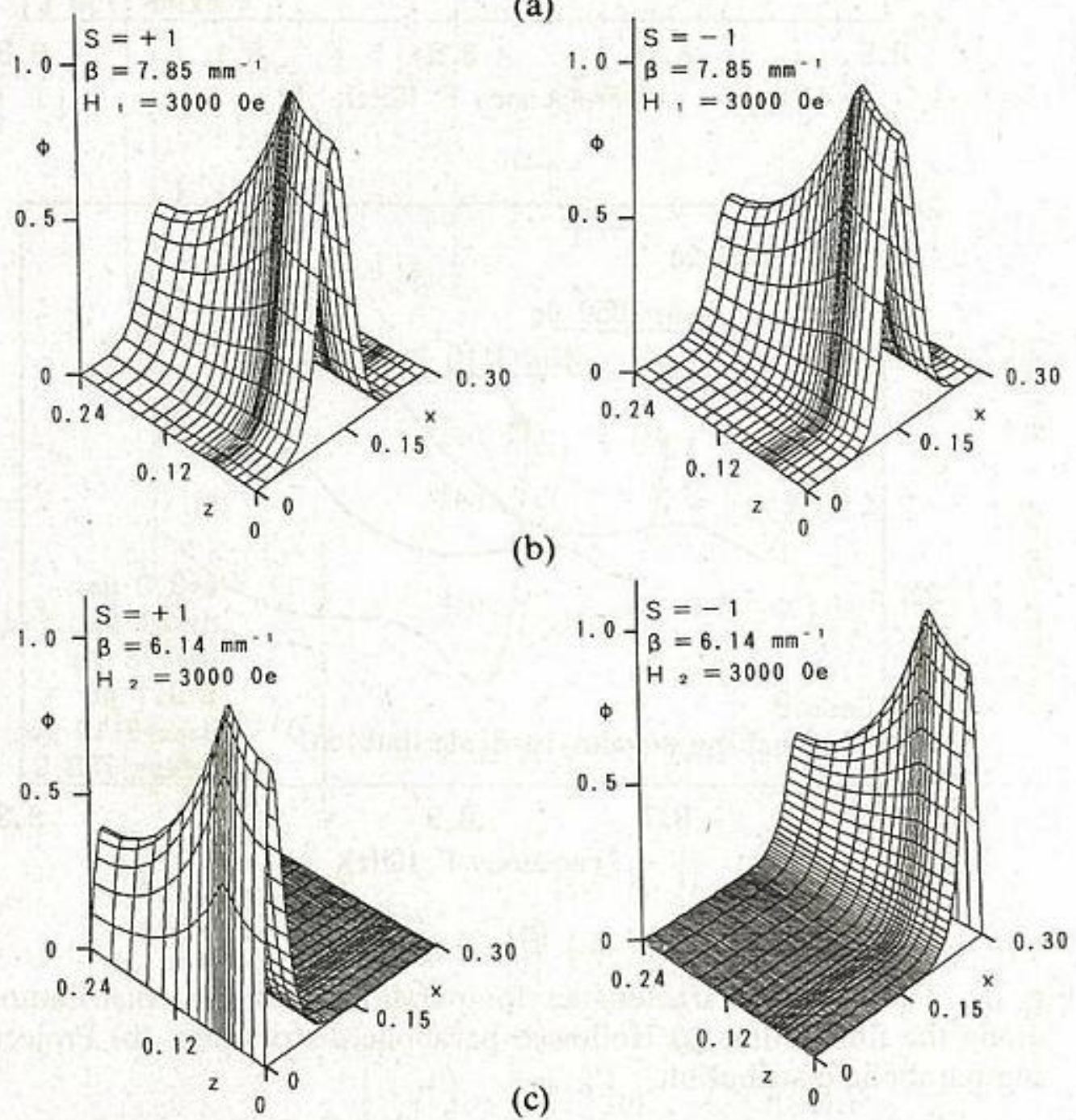

(c)

Fig. 7. Potential profiles. (a) Homogeneous distribution. (b) Hollowed parabolic distribution. (c) Projecting parabolic distribution.

manner. In order to examine the validity of the present method, MSW modes in a YIG-loaded rectangular waveguide and in a YIG film of finite width are calculated. We also present the application of this approach by analyzing the MSFVW propagation in a YIG film with the bias field of parabolic distribution along the film width. It is found that the parabolic bias field offers an improvement in the nondispersive bandwidth.

\section{REFERENCES}

[1] F. R. Morgenthaler, "Magnetostatic waves bound to a DC field gradient," IEEE Trans. Magn., vol. MAG-13, pp. 1252-1254, Sept. 1977.

[2] T. W. O'Keeffe and R. W. Patterson, "Magnetostatic surface-wave propagation in finite samples," J. Appl. Phys., vol. 49, pp. 4886-4895, Sept. 1978.

[3] F. R. Morgenthaler, "Bound magnetostatic waves controlled by field gradients in YIG single crystal and epitaxial films," IEEE Trans. Magn., vol. MAG-14, pp. 806-810, Sept. 1978.

[4] S. N. Bajpai and N. C. Srivastava, "Magnetostatic bulk wave propagation in multilayered structure," Electron. Lett., vol. 16, pp. 269-270, Mar. 1980.

[5] M. Tsutsumi, Y. Masaoka, T. Ohira, and N. Kumagai, "A new technique for magnetostatic wave delay lines," IEEE Trans. Microwave Theory Tech., vol. MTT-29, pp. 583-587, June 1981.
[6] F. R. Morgenthaler, "Nondispersive magnetostatic forward volume waves under field gradient control," J. Appl. Phys., vol. 53, pp. 2652-2654, Mar. 1982.

[7] N. P. Vlannes, "Investigation of magnetostatic waves in uniform and nonuniform magnetic bias fields with a new induction probe," presented at Third Joint Intermag-Magnetism and Magnetic Materials Conf., Montreal, Que., Canada, July 1982.

[8] D. D. Stancil and F. R. Morgenthaler, "Guiding magnetostatic surface waves with nonuniform in-plane fields," J. Appl. Phys., vol. 54, pp. 1613-1618, Mar. 1983.

[9] F. R. Morgenthaler, "Control of magnetostatic waves in thin films by means of spatially nonuniform bias fields," Circuits Syst. Signal Process., vol. 4, pp. 63-88, 1985.

[10] M. Radmanesh, C. M. Chu, and G. I. Haddad, "Magnetostatic wave propagation in a finite YIG-loaded rectangular waveguide," IEEE Trans. Microwave Theory Tech., vol. MTT-34, pp. 1377-1381, Dec. 1986.

[11] M. Radmanesh, C. M. Chu, and G. I. Haddad, "Magnetostatic waves in a normally magnetized waveguide structure," IEEE Trans. Microwave Theory Tech., vol. MTT-35, pp. 1226-1229, Dec. 1987.

[12] D. D. Stancil, "Variational formulation of magnetostatic wave dispersion relations," IEEE Trans. Magn., vol. MAG-19, pp. 1865-1867, Sept. 1983.

[13] E. Sawado and N. S. Chang, "Variational approach to analysis of propagation of magnetostatic waves in highly inhomogeneously magnetized media," J. Appl. Phys., vol. 55, pp. 1062-1067, Feb. 1984 .

[14] Y. Long, M. Koshiba, and M. Suzuki, "Finite-element solution of planar inhomogeneous waveguides for magnetostatic waves," IEEE Trans. Microwave Theory Tech., vol. MTT-35, pp. 731-736, Aug. 1987.

[15] P. E. Lagasse, "Higher-order finite-element analysis of topographic guides supporting elastic surface waves," J. Acoust. Soc. Amer., vol. 53, pp. 1116-1122, Apr. 1973.

[16] O. C. Zienkiewitz, The Finite Element Method, 3rd ed. London: McGraw-Hill, 1977.

\section{幽}

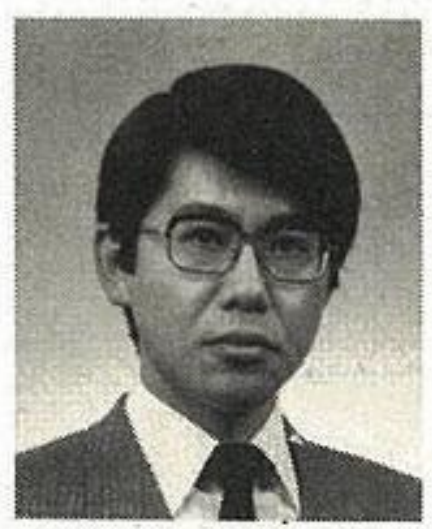

Masanori Koshiba (SM'84) was born in Sapporo, Japan, on November 23, 1948. He received the B.S., M.S., and $\mathrm{Ph} . \mathrm{D}$. degrees in electronic engineering from Hokkaido University, Sapporo, Japan, in 1971, 1973, and 1976, respectively.

In 1976, he joined the Department of Electronic Engineering, Kitami Institute of Technology, Kitami, Japan. From 1979 to 1987, he was an Associate Professor of Electronic Engineering at Hokkaido University, and in 1987 he became a Professor. He has been engaged in research on lightwave technology, surface acoustic waves, magnetostatic waves, microwave field theory, and applications of finite-element and boundaryelement methods to field problems.

Dr. Koshiba is a member of the Institute of Electronics, Information and Communication Engineers (IEICE), the Institute of Television Engineers of Japan, the Institute of Electrical Engineers of Japan, the Japan Society for Simulation Technology, and the Japan Society for Computational Methods in Engineering. He received the Paper Award in 1987 from the IEICE.

\section{承}

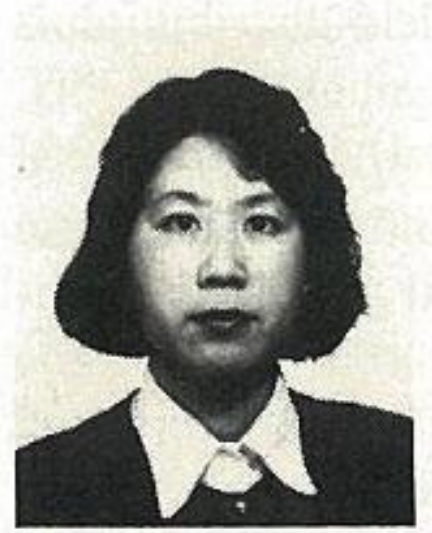

Yi Long was born in Nanchang, China, on December 1, 1955. She received the B.S. and M.S. degrees in electronic engineering from Huazhong University of Science and Technology, Wuhan, China, in 1982 and 1984, respectively, and the $\mathrm{Ph} . \mathrm{D}$. degree in electronic engineering from Hokkaido University, Sapporo, Japan, in 1989. 